Case Report

\title{
Sarcoid Like Reaction Confounding the Diagnosis of Pulmonary Adenocarcinoma
}

\author{
Naga Srinivas Sirikonda*, Abdulmonam Ali \\ Department of Pulmonary and Critical Care Medicine, Good Samaritan Hospital, Mount Vernon, USA \\ Email address: \\ naga.sirikonda@yahoo.com (N. S. Sirikonda) \\ ${ }^{*}$ Corresponding author
}

\section{To cite this article:}

Naga Srinivas Sirikonda, Abdulmonam Ali. Sarcoid Like Reaction Confounding the Diagnosis of Pulmonary Adenocarcinoma. American Journal of Internal Medicine. Vol. 7, No. 5, 2019, pp. 124-126. doi: 10.11648/j.ajim.20190705.13

Received: August 29, 2019; Accepted: September 21, 2019; Published: October 10, 2019

\begin{abstract}
Sarcoidosis and other forms of co-existing granulomatous inflammation have been previously reported to occur along with bronchogenic carcinoma although rare. In addition, sarcoidosis has been reported as an independent risk factor for development of lung cancer. The histological findings of granulomatous inflammation can be misleading in patients with lung nodules and especially with a high pretest probability of lung cancer. We report a case of middle age Caucasian female with smoking history and obstructive lung disease who presented with multiple spiculated pulmonary nodules in both upper lobes. Pretest probability of lung cancer was high in this patient because of smoking history, location (upper lobe), appearance (spiculation) and abnormal Positron Emission Tomography (PET) scan. Initial needle biopsy by interventional radiologist revealed non-caseating granulomatous inflammation without any malignant cells leading to diagnosis of sarcoidosis. But patient underwent surgical lung biopsy because of no response to steroids that revealed the diagnosis of adenocarcinoma. Surgical lung biopsies also revealed coexisting granulomatous inflammation in the vicinity of the malignancy changes. It is difficult to establish whether the sarcoid finding in this case is an immunogenic reaction to malignancy or a precursor for malignancy. Sarcoid like reaction can rarely be seen in bronchogenic carcinoma misleading the diagnosis at times.
\end{abstract}

Keywords: Bronchogenic Carcinoma, Granulomatous Inflammation, Immunological Reaction, Lung Nodules, Malignancy, Pulmonary Adenocarcinoma, Sarcoid Reaction

\section{Introduction}

This case report discusses about a patient with extensive smoking history and multiple spiculated pulmonary nodules in Computed tomography (CT) of chest. Initial CT guided biopsy showed non caseating granulomatous inflammation without any malignant cells leading to the diagnosis of sarcoidosis by exclusion but did not respond to steroid treatment. Further evaluation by thoracic surgery and video assisted thoracoscopic wedge lung biopsy showed pulmonary adenocarcinoma along with granulomatous inflammation (sarcoid like reaction). There was a delay in the final diagnosis of malignancy for this patient because of confounding histologic findings of sarcoid like reaction in the initial biopsies and hence we recommend further invasive diagnostic procedures to rule out malignancy in such cases especially with high pretest probability of carcinoma.
Sarcoidosis is a granulomatous disease of unknown etiology mainly involving lungs and lymph nodes in the mediastinum and hilum [1]. First line treatment of sarcoidosis is corticosteroids with antimetabolites generally used when unresponsive to steroids [1]. Granulomatous inflammation can rarely be seen in lung cancer tumors and regional draining lymph nodes which is called 'sarcoid like reaction' as they do not fulfill the criteria for systemic sarcoidosis [2]. Sarcoid like reaction can coexist with malignancy either at the time of diagnosis or later during follow up after treatment or from oncologic immunotherapy $[2,10,14]$. It can be challenging at times in such cases when tissue biopsies only pick up sarcoid changes without revealing malignancy because of sampling areas in that particular tumor and further delaying the actual diagnosis which happened in our case. 


\section{Case Report}

A 66 year old Caucasian female with history of moderate COPD, asthma and fifty pack year smoking had low dose CT chest for lung cancer screening that showed three spiculated nodules in left upper lobe measuring $25 \mathrm{~mm}, 18 \mathrm{~mm}, 8 \mathrm{~mm}$ and one $12 \mathrm{~mm}$ spiculated nodule in right upper lobe along with emphysematous changes (Figure 1). The $25 \mathrm{~mm}$ left upper lobe lesion had central cavitation. No mediastinal and hilar adenopathy was seen. PET scan showed intense hypermetabolic activity in $18 \mathrm{~mm}$ left upper lobe nodule and mild to moderate hypermetabolic activity in the other lung nodules (Figure 2). No hypermetabolic activity was seen in mediastinal or hilar lymph nodes or extrathoracic regions.
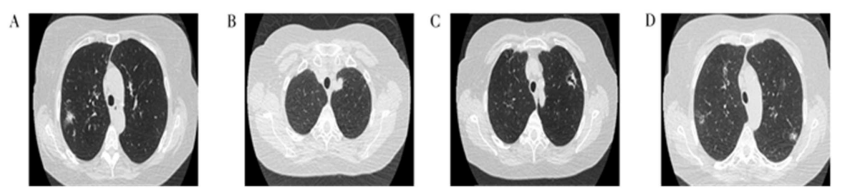

Figure 1. Multiple spiculated nodules in right and left upper lobes of lungs (A) Low dose CT chest showing $12 \times 11 \mathrm{~mm}$ spiculated nodule in right upper lobe (B) Low dose CT chest showing spiculated $18 \times 12 \mathrm{~mm}$ nodule in medial left upper lobe $(C)$ Low dose CT chest showing $25 \times 25 \mathrm{~mm}$ cavitary lesion in left upper lobe (D) Low dose CT chest showing $8 \times 4 \mathrm{~mm}$ spiculated nodule in left upper lobe.
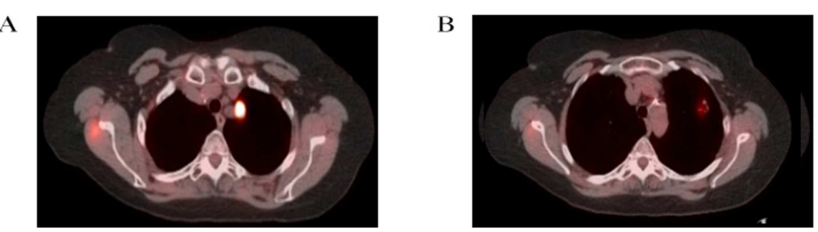

Figure 2. (A) PET scan showing intensely hypermetabolic medial left upper lobe nodule with SUV 13.4 (B) PET scan showing mild to moderately hypermetabolic left upper lobe cavitary lesion with SUV 3.2.

Based on the Swensen/Mayo nodule malignancy risk calculator, the patient's lung cancer risk was estimated to be $82 \%$. CT guided biopsy of the left upper lobe $25 \mathrm{~mm}$ cavitary nodule showed non-caseating granulomatous inflammation (Figure 3). Silver stain showed rare budding yeast forms later determined to be histologic contaminants. Acid Fast Bacilli stain showed no acid fast bacilli. Tissue cultures showed no growth of fungus or acid fast bacillus and fungal serologies were also negative. After ruling out non-infectious granulomatous lung diseases such as hypersensitivity pneumonitis, vasculitis and rheumatoid arthritis, the patient was started on systemic steroids for possible sarcoidosis.

Repeat CT chest in two months showed stable nodules without significant change in size. There were no new nodules or lymphadenopathy. Steroids were continued. Follow up CT chest three months later showed increase in the size of the nodules. Since there was no response to steroids, the patient was referred to Thoracic surgeon for wedge biopsy. VATS wedge resection of the two nodules in the left upper lobe was performed and the biopsies showed moderately differentiated invasive adenocarcinoma with a predominant lepidic pattern (Figure 4). Granulomatous inflammation was again seen in the center of these malignant nodules. EGFR and ALK mutations were negative. Patient also underwent CT guided needle biopsy of the right upper lobe nodule for staging, which also showed moderately differentiated adenocarcinoma. She was diagnosed with stage IV (T1c, N0, M1a) pulmonary adenocarcinoma and started on carboplatin, pemetrexed and bevacizumab chemotherapy. Repeat CT chest in two months after starting chemotherapy showed improvement in the size of both left upper lobe and right upper lobe nodules.
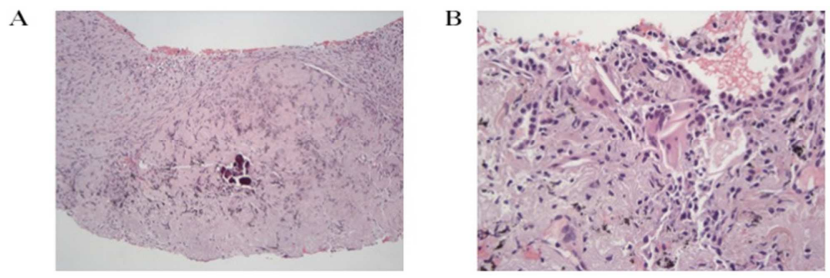

Figure 3. Histopathological examination (H/E staining) of CT guided core biopsy of $25 \mathrm{~mm}$ Cavitary, lateral left upper lobe nodule (A) Well circumscribed, poorly formed granuloma with central calcification. No malignant cells were identified (B) High-power magnification showing lymphocytic inflammation surrounding multinucleated, Langerhans-type histiocytes and reactive alveolar spaces.
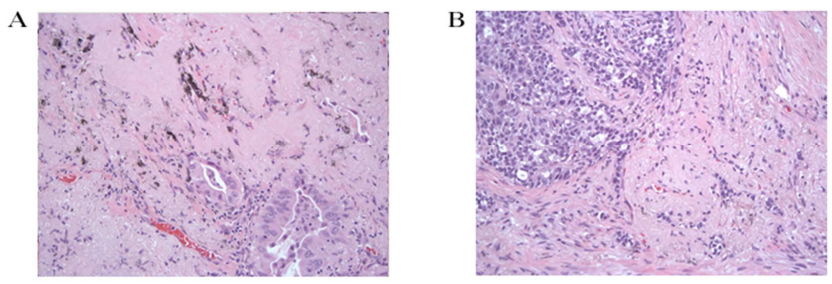

Figure 4. Histopathological examination of wedge biopsies (Wedge of apical $12 \mathrm{~mm}$ left upper lobe nodule and Wedge of posterior $8 \mathrm{~mm}$ LUL nodule respectively) HPE examination of both nodules showed adenocarcinoma with distinct morphologic features. Both tumors were associated with hyalinizing fibrosis and chronic inflammation which may be confused with granulomatous inflammation if inadequately sampled.

\section{Discussion}

Sarcoidosis and lung cancer are the most common lung diseases. Lung cancer is the second most common cancer in both men and women with $14 \%$ of all new cancers being lung cancer. Lung cancer is also the leading cause of cancer death in both gender [3]. Hence delay in the lung cancer diagnosis can lead to increased morbidity and mortality. Sarcoidosis is a multisystem granulomatous disease of unknown etiology that primarily affects the lungs and lymphatic system in more than $90 \%$ of patients. Most common imaging findings of sarcoidosis include mediastinal and hilar lymphadenopathy and lung nodules, which are also seen in bronchogenic carcinoma $[4,5]$. PET scan usually reveals similar findings in both diseases showing increased hypermetabolic activity in lung nodules and lymph nodules. Definitive diagnosis of sarcoidosis and lung cancer is usually accomplished by histological demonstration of noncaseating granulomas and malignant cells respectively. However diagnostic dilemma can arise every now and then when the sarcoid like reaction accompanies the malignant tissue in bronchogenic carcinoma.

Sarcoidosis or sarcoid like reaction can rarely coexist in the 
lung cancer cases. Granulomatous inflammation has been reported to be seen in tumor nodules and within regional lymph nodes at the time of lung cancer diagnosis in some cases [6-9]. Wolbech et al first described sarcoid like reactions in two autopsy cases of carcinoma of colon and jaw [15]. A case of small cell lung cancer in lung nodule with sarcoid like reaction in mediastinal and hilar lymph nodes causing diagnostic dilemma regarding lymph node metastasis has been reported [14]. Tan et al reported a case with lung adenocarcinoma who underwent lobectomy and 4 years later developed new mediastinal and hilar adenopathy that revealed sarcoid like reaction in Endobronchial ultrasound (EBUS) guided fine needle aspiration [10]. Bronchogenic carcinoma was also reported to occur in patients with previous diagnosis of sarcoidosis [7, 8, 13]. In a retrospective study based on Danish registry in 1973, sarcoidosis was associated with three fold increased incidence of bronchogenic cancer and malignant lymphoma. The cancer incidence was higher in symptomatic sarcoidosis (1-5 times) patients compared to asymptomatic sarcoidosis (1-2 times) [12].

The causal relationship between sarcoidosis and bronchogenic carcinoma is unclear. There have been several hypothesis concerning their cause and effect relationship. It has been postulated that fibrous tissue and cell mediated immune abnormalities in sarcoidosis can predispose to lung cancer [8]. Oncocentric theory postulates sarcoidosis as an immunological reaction to tumor antigen [11].

Our patient had granulomatous inflammation in the lung nodules in the initial CT guided biopsy and also the central part of the VATS wedge biopsies but the malignancy was diagnosed in the wedge biopsy only. It is possible that the malignant cells were missed in the initial needle core biopsy as the specimen may have been obtained from the center of the nodule. Sarcoid like reaction in the center of the nodules is likely secondary to immunologic reaction. The patient was misdiagnosed with sarcoidosis based on the initial biopsy and the cancer diagnosis was delayed for few months because of the confounding biopsy results with granulomatous inflammation associated with malignancy. We suggest pursuing further diagnostic procedures like VATS in cases like this with high pretest probability of malignancy even though the core needle or bronchoscopy biopsies show the granulomatous inflammation. The nonsurgical biopsies may only show the granulomatous inflammation without capturing the malignant cells in cases like this with coexistent malignancy and sarcoid like reaction and our case is a good example for this.

\section{Conclusion}

Lung cancer and sarcoidosis are the most common pulmonary diseases. Sarcoidosis is a benign inflammatory disease that responds to steroids, whereas lung cancer has multidisciplinary treatment approach (surgery, chemotherapy, radiation) depending the staging. Both diseases can have similar imaging findings and definitive diagnosis is established by tissue sampling. Diagnostic dilemma can arise when sarcoid like reaction (granulomatous reaction) accompany the malignancy as a result of immunological reaction to tumor antigen. Less invasive tissue sampling procedures like core needle biopsy and bronchoscopy assisted biopsy at times may not reveal the accurate diagnosis in such cases. We suggest pursuing more invasive techniques like surgical lung biopsies in cases with sarcoid like findings yet has a high clinical suspicion of malignancy.

\section{References}

[1] Paolo Spagnolo MD et al. Pulmonary Sarcoidosis. The Lancet Respiratory Medicine. Volume 6, ISSUE 5, P389-402, May 01, 2018.

[2] Spiekermann, C., Kuhlencord, M., Huss, S., Rudack, C., Weiss, D."Coexistence of sarcoidosis and metastatic lesions: A diagnostic and therapeutic dilemma (Review)". Oncology Letters 14, no. 6 (2017): 7643-7652.

[3] Key statistics for Lung cancer. American Cancer Society. https://www.cancer.org/cancer/non-small-cell-lung-cancer/abo ut/key-statistics.html.

[4] Baughman RP1, Culver DA, Judson MA. A concise review of pulmonary sarcoidosis. Am J Respir Crit Care Med. 2011 Mar 1; 183 (5): 573-81.

[5] Hilario Nunes et al. Imaging of sarcoidosis of the airways and lung parenchyma and correlation with lung function. European Respiratory Journal 2012 40: 750-765.

[6] Joon Seon Song, Seungkoo Lee, Yong Hee Kim et al. Sarcoidosis Associated with Adenocarcinoma of the Lung. The Korean Journal of Pathology 2008; 42: 108-12.

[7] Kachalia AG, Ochieng P, Kachalia K, Rahman H. Rare coexistence of sarcoidosis and lung adenocarcinoma. Respir Med Case Rep. 2014 Mar 15; 12: 4-6.

[8] Yamasawa H1, Ishii Y, Kitamura S. Concurrence of sarcoidosis and lung cancer. A report of four cases. Respiration. 2000; 67 (1): 90-3.

[9] Laurberg P. Sarcoid reactions in pulmonary neoplasms. Scand J Respir Dis. 1975 May; 56 (1): 20-7.

[10] QL Tan et al. Sarcoid-like reaction associated with lung adenocarcinoma: A case report. SAGE Journal. 2019, Vol. 28 (1) 68-70.

[11] Reich JM. Neoplasia in the etiology of sarcoidosis. Eur J Intern Med. 2006 Mar; 17 (2): 81-7.

[12] Brincker H, Wilbek E. The incidence of malignant tumours in patients with respiratory sarcoidosis. Br J Cancer. 1974 Mar; 29 (3): 247-51.

[13] Sakula A. Bronchial Carcinoma and Sacoiodosis. Br J Cancer. 1963 Jun; 17: 206-12.

[14] Ramadas et al. Lung cancer or sarcoidosis: A diagnostic dilemma. Journal of Cancer Therapeutics \& Research ISSN 2049-7962|Volume 5|Article 2. 2016.

[15] Wolbach, SB. A new type of cell inclusion, not parasitic, associated with disseminated granulomatous lesions. J Med Res 1911; 24: 243-261. 